\title{
Der Ressourcen-Nexus als Frühwarnsystem für zukünftige zwischenstaatliche Konflikte
}

\author{
Raimund Bleischwitz
}

Online publiziert: 23. Dezember 2014

(C) Die Autor(en) 2014. Dieser Artikel ist auf Springerlink.com mit Open Access verfügbar.

Zusammenfassung: Der Beitrag analysiert den Ressourcen-Nexus, das heißt das Wirkungsgeflecht zwischen Nutzungsformen natürlicher Ressourcen, als Thema der Außen- und Sicherheitspolitik. Er diskutiert das Zusammenwirken von Energie, Wasser, Nahrungsmitteln, Land und mineralischen Rohstoffen im Lichte der aktuellen Debatten und analysiert mögliche Konflikte und Chancen, die sich daraus ergeben. Diese Debatte gewinnt Einfluss, weil sie Akteure aus unterschiedlichen Politikbereichen zusammenführt. Der Artikel entwickelt erste Schritte, wie diese Thematik angegangen werden kann und wie ein Frühwarnsystem aussehen könnte. Deutschland könnte im Zuge einer wachsenden Verantwortung eine internationale Initiative zum Ressourcen-Nexus initiieren.

Schlüsselwörter: Ressourcen-Nexus · Umwelt · Energie $\cdot$ Sicherheit

\section{The Resource Nexus as a Risk Radar for Future interstate Conflics}

Abstract: The article analyses the resource nexus, i.e. interlinkages among the resources energy, water, food, land, and minerals as a challenge for foreign policy. Reviewing recent debates it underlines both potential conflicts and opportunities. The debate is gaining ground as many actors from a variety of policy areas express

Der Autor dankt Maria Behrens und Rüya Perincek für nützliche Hinweise und Rückmeldungen zu einem Entwurf. Hilfreich waren ferner laufende Diskussionen mit Philip Andrews-

Speed, Tim Boersma, Holger Hoff, Corey Johnson, Geoffrey Kemp, Rabi Mohtar, und Stacy VanDeveer sowie Workshops mit dem UNEP International Resource Panel (7.-8.10.2014) und am Royal United Services Institute for Defence and Security Studies (RUSI, 9.10.2014).

Prof. Dr. R. Bleischwitz $(\bowtie)$

UCL Institute for Sustainable Resources,

14 Upper Woburn Place,

WC1H 0NN London, Vereinigtes Königreich

E-Mail: r.bleischwitz@ucl.ac.uk 
stakes in it. The article derives steps on encountering the challenges ahead and develops elements of a risk radar. Germany may accept international responsibility and initiate an international forum on the resource nexus.

Keywords: Resource nexus $\cdot$ Environment $\cdot$ Energy $\cdot$ Security

\section{Einleitung}

Die weltweite Nachfrage nach Rohstoffen hat historische Höchstmarken erreicht. Der herkömmliche Blick auf einzelne Rohstoffe ist jedoch verengt. Die Nutzung natürlicher Ressourcen ist durch dynamische Wechselwirkungen untrennbar miteinander verbunden. Während bis zu Beginn der industriellen Revolution mehr Stoffe durch die Natur als vom Menschen bewegt wurden, ist die Weltwirtschaft mit dem Wachstum der Schwellenländer spätestens seit den neunziger Jahren zu einer geologischen Urgewalt geworden. Sie nimmt mehr als die Hälfte der Photosynthese der Natur in Anspruch, verändert sichtbar jede Landschaft und die Wasserläufe auf der Erde, und greift in sensible ökologische Regelungsmechanismen ein (Steffen et al. 2011; Rockström et al. 2009).

Das Anliegen dieses Beitrags liegt darin, diesen Ressourcen-Nexus als Frühwarnsystem für potenzielle Konflikte zu analysieren und die Rolle der Außen- und Sicherheitspolitik zu thematisieren. Unser Beitrag soll die Debatte über den „Kampf um Rohstoffe" differenzieren. Diese Debatte verläuft typischerweise über den Zugang zu einem Rohstoff (z. B. Erdöl) und thematisiert entsprechende Verteilungskonflikte. Wir möchten darstellen, dass neuartige Konfliktspiralen aus den Wechselwirkungen der Ressourcennutzung entstehen können, wo Ressourcenkonflikte jeweils einzeln betrachtet beherrschbar erscheinen, jedoch in ihren kumulativen Wirkungen eskalieren können. Wir nennen dies den Ressourcen-Nexus. Unsere These dazu lautet, dass die sozio-ökonomische und politische Dynamik der Wechselwirkungen zwischen Energie, Wasser, Nahrungsmitteln und anderen Ressourcen ein enormes Konfliktpotential birgt, das über so genannte Konfliktmineralien, andere Einzelstoffe und direkte umweltbezogene Wirkungen hinausgeht. Mit einer klugen Politik und neuen Governance-Ansätzen kann es aber auch pragmatische Kooperationschancen und den Aufbau nachhaltiger Märkte geben.

Die aktuellen Konflikte im Nahen Osten können demnach ohne eine Analyse des Ressourcen-Nexus weder verstanden noch einer Lösung näher gebracht werden. Erdöl, Wasser und Nahrungsmittel sind untrennbar miteinander verbunden: Die Wasserknappheit verlangt nach neuer Energie für Pumpsysteme und Meerwasserentsalzung, und es macht einen Unterschied, ob Kernkraftwerke und Staudämme errichtet werden oder Solarenergie erzeugt wird. Brotpreise können dann als soziales Sprengmittel wirken. Es entstehen viele Einzelfeuer, die sich zu einem Flächenbrand verbinden.

Die Rede von Bundespräsident Gauck bei der Münchener Sicherheitskonferenz 2014 lief unter der Überschrift, dass Deutschland bereit sein muss, mehr zu tun. Nach unserer Sicht sollte dies für die Risiken des Ressourcen-Nexus und das Engagement Deutschlands als industriebasierter Handelsnation mit einer ambitionierten Umwelt- 
politik in besonderem Maße gelten. Neben möglichen Konflikten gilt es auch Interdependenzen zu beachten: Während Industrieländer Interesse am Zugang zu Rohstoffen haben, ist die Umweltsituation in Anbieterländern oft katastrophal und sie haben Interesse an Know-how, Technologien und entsprechenden Umwelt-Innovationen. Ein ganzheitlicher Ansatz, der Ressourcen, Umwelt und Entwicklung miteinander verbindet, bietet insofern Vorteile.

Unser Beitrag skizziert im Folgenden zunächst die Debatte über den Ressourcen-Nexus. Er analysiert anschließend die Konfliktmechanismen. In einem weiteren Kapitel werden Kooperationschancen ausgelotet. Schließlich entwerfen wir erste Schritte zu einem Frühwarnsystem und ziehen Schlussfolgerungen für die Außenund Sicherheitspolitik.

\section{Die Debatte über den Ressourcen-Nexus}

Der Ressourcen-Nexus entsteht aus dem Wirkungsgeflecht der Inanspruchnahme von Energie, Nahrungsmitteln, Wasser, Land und mineralischen Rohstoffen. Er bedeutet, dass Handlungen von Akteuren, die auf die Nutzung einer Ressource ausgerichtet sind, immer auch Konsequenzen für andere Ressourcen und deren Nutzung haben.

Der Ressourcen-Nexus hat seinen Ausgangspunkt in natürlichen Inputs für wichtige Produktionssysteme. Kraftwerke zur Energieversorgung sind auf Kühlwasser angewiesen; Wasserversorgungssysteme sind energieintensiv. Typischerweise werden Systeme der Energie- und Wasserversorgung jedoch geplant und betrieben, ohne derartige Wechselwirkungen abzuschätzen. Die Nutzungskonflikte zwischen dem rapide wachsenden Bedarf nach Pflanzen für eine alternative Energieerzeugung und Getreide haben zu sprunghaften Preissteigerungen für Nahrungsmittel geführt. Der Nexus zwischen Energie, Wasser und agrarischen Rohstoffen ist insofern offensichtlich. Aber auch der Bezug zu Land, das als Anbaufläche und ökologischer Regelungsmechanismus essenziell ist, sollte Beachtung finden. Und mineralische Rohstoffe sind als Düngemittel und materielles Rückgrat der Energie- und Wasserversorgung ebenso bedeutsam wie kritische Materialien für die modernen Umwelttechnologien und eine saubere Energieversorgung. Für mineralische Rohstoffe gilt, dass der Bergbau und nachgelagerte Verarbeitungsstufen energie- und wasserintensiv sind.

Auf der systemischen Ebene sind es also Abhängigkeiten von Produktionsinputs natürlicher Ressourcen. Diese Inputs werden in der Regel von anderen Unternehmen bzw. von Märkten geliefert. Weil es sich um natürliche Ressourcen handelt, die aus Ökosystemen entnommen werden, können bei sprunghaften Nachfrageänderungen jedoch Nachhaltigkeitsfallen entstehen. Die Inputs von Wasser und von biotischen Stoffen entstammen unmittelbar dem Naturhaushalt; ihre Übernutzung hat unmittelbar negative Umweltauswirkungen. Bei anderen Inputs sind es Landschaftseingriffe und andere Formen der Umweltbelastungen, die im Wirkungsgeflecht zu Störungen führen können. Anders gesagt bringen Nachfrageänderungen die ökonomische Bereitstellungsfunktion von Ökosystemen in Konflikte mit ihrer Regelungs- und Stabilisierungsfunktion. 
Charakteristisch für das Wirkungsgeflecht im Ressourcen-Nexus sind die folgenden Merkmale:

1. Die Geographie der Wirkungen ist oft lokal und regional. Sie kann sich jedoch aufgrund des internationalen Handels oder aufgrund von weltweit vernetzten Ökosystemen auch sehr rasch und intensiv in entfernten Gegenden zeigen. Fernwirkungen treten in Form lokaler Verknappungen auf, Beeinträchtigungen der natürlichen Umwelt oder schwerwiegenden Störungen von sozialen Lebensbedingungen. Mittlerweile gilt als belegt, dass die Dürre in der nordchinesischen Weizenanbauregion der Provinzen Henan und Anhui im Winter 2011 als Mitauslöser für die politischen Umwälzungen in Ägypten und im „Arabischen Frühling“ anzusehen ist, da die weltweiten Weizenpreise deutlich anzogen und die rapide steigenden Brotpreise soziale Unruhen hervorriefen (Breisinger et al. 2011; Sternberg 2012).

2. Sozio-Ökonomie und Politik führen zu Multiplikatoreffekten. Die Wirkungen von Preiserhöhungen aufgrund von Verknappungen potenzieren sich durch Importabhängigkeiten, soziale Instabilitäten, Markteingriffe in Form von Subventionen, Exportrestriktionen und Spekulation, sowie durch andere Faktoren. Der entscheidende Punkt ist hier, dass diese Dimension von Umweltsystemanalysen übersehen wird und in Nexusanalysen einbezogen werden muss.

3. Der unterschiedlich lange Lebenszyklus von Ressourcen erhöht die Komplexität. Er umfasst das primäre Angebot aufgrund von geologischen und naturräumlichen Gegebenheiten sowie die Phasen von Produktion, Nutzung und eine potenzielle Wiedernutzung. Bei Nahrungsmitteln ist dieser Lebenszyklus deutlich kürzer als z. B. bei Metallen, Baustoffen und anderen Ressourcen, deren Lebensdauer und deren Potenziale zur Rückgewinnung hoch sind. Viele Akteure fokussieren sich auf das primäre Angebot und unterschätzen die Chancen der Ressourceneffizienz - verstanden als die verbesserte Nutzung aller Ressourcen entlang ihres Lebenszyklus und in internationalen Wertschöpfungsprozessen. Diese verkürzte Sicht führt zu einer überdimensionierten Planung und Fehleinschätzungen über Bedarf. Sie unterstützt so Konflikte über den Zugang zu primären Ressourcen, aber auch Konflikte um Abfall und Sekundärrohstoffe.

4. Nicht-intendierte Wirkungen spielen eine erhebliche Rolle. Hat die politisch gut gemeinte Unterstützung der Biokraftstoffe die Preiserhöhungen für Nahrungsmittel vorausgesehen oder gar bezweckt? Sicher nicht. Weil Planung, Management und Politik in der Regel entlang etablierter Zwecke und Funktionen organisiert sind, multiplizieren sich nicht-intendierte Wirkungen. Die strafrechtliche Diskussion, ob jemand bestimmte Folgen billigend in Kauf nimmt oder gar fahrlässig handelt, dürfte insofern neuen Interpretationen entgegen sehen. Aber sogar für vorsorgeorientierte und kooperationswillige Akteure ist der Ressourcen-Nexus eine Herausforderung bezüglich der Governance-Mechanismen.

Das Beispiel der Staudammplanung in China kann diese Merkmale verdeutlichen: China benötigt zusätzlichen Strom zur Bewältigung seiner wachsenden Güterproduktion, die in nennenswerten Teilen in die westliche Welt geht. Ein großer Teil der Stromproduktion soll durch Wasserkraft erzeugt werden. Für die großen Staudammprojekte wurde eine angebotsorientierte Planung zugrunde gelegt. Als Folge werden 
mehr als zwanzig Megaprojekte geplant, für deren Bau Millionen von Menschen umgesiedelt und Tonnen an Materialien benötigt werden, einschließlich kritischer Metalle zur Regeltechnik. Für die Produktion der Baustoffe wird wiederum Energie benötigt, für die Kohle importiert wird. Staudammprojekte haben insofern nicht nur erhebliche ökologische und sozio-ökonomische Wirkungen in der Region, sondern auch Auswirkungen auf internationaler Ebene. Politisch kommt verschärfend hinzu, dass die Nachbarstaaten in Asien mit Misstrauen die Veränderung ihrer Flussoberläufe beobachten und die Tatsache missbilligen, dass China sich bislang internationalen Wasserabkommen verschließt.

Als Ausgangspunkt der Debatten über den Ressourcen-Nexus können die BonnKonferenz 2011 (Hoff 2011) und Publikationen u. a. des niederländischen Umweltamts (Slingerland et al. 2011), der Transatlantic Academy (Andrews-Speed et al. 2012, 2014), des europäischen Entwicklungsberichts (European Commission 2012), und von Chatham House (Lee at al. 2012) angesehen werden. Diese stellen insbesondere den Nexus zwischen Wasser - Nahrungsmittel - Energie in den Mittelpunkt (aber auch andere Ressourcen), sie betonen die Sicherheitsdimension und die gegenseitige Abhängigkeit von Umwelt und Entwicklung. Vor allem aber liefern sie eine Reihe überzeugender Fallstudien über das Wirkungsgeflecht im Ressourcen-Nexus und dessen Implikationen für Politik, Märkte und Sicherheit. Eine Reihe von wissenschaftlichen Publikationen hat dies weiter fundiert (Bazilian et al. 2011; Lawford et al. 2013); für die Wasserabhängigkeit der Stromversorgung (Ackerman und Fisher 2013), in Bezug auf Land (Ringler et al. 2013), mineralische Ressourcen (Giurco et al. o. J.), Nährstoffe (Mo und Zhang 2013), und regionale Fallstudien für Südasien (Mukherji 2007; Rasul 2014), Nordafrika und den Nahen Osten (Siddiqi und Anadon 2011).

Die Denkanstöße zum Ressourcen-Nexus haben relativ rasch politischen Einfluss gezeigt: Die asiatische Wirtschaftsorganisation der Vereinten Nationen (UNESCAP 2013) stellt den Ressourcen-Nexus in den Mittelpunkt einer Analyse. Der National Intelligence Council der USA, das oberste Gremium zur Koordination der US Geheimdienste, warnt vor den Sicherheitsrisiken, die aus dem Ressourcen-Nexus resultieren können und nennt beispielsweise die grenzüberschreitenden Flüsse in Asien, Amu Darya und Brahmaputra, sowie die angespannte Situation in Pakistan (NIC 2012). Seit Januar 2014 debattiert der US-Senat über den Wasserverbrauch der Energiewirtschaft im Zusammenhang mit der monatelangen Dürre in Teilen der USA als Nexus of Energy and Water for Sustainability (NEWS) Act of 2014 (S. 1971) (Murkowski-Wyden-Bill). In Indien lassen sich ähnliche Debatten und Politikansätze identifizieren (Ghosh 2013). Unterstützende Arbeiten gibt es in der Internationalen Energieagentur, von einem Unternehmsforum (WBCSD 2012) und in der Welternährungsorganisation (FAO) (Giampietro et al. 2013).

Die Debatten über den Ressourcen-Nexus sind also dadurch geprägt, dass viele Akteure aus „harten“ Politikbereichen wie Sicherheit, Wirtschaft und Finanzen und aus ,, weichen " Politikbereichen wie Umwelt, Entwicklung und Soziales den Nexus zu ihrem Anliegen gemacht haben. Dies ist ein deutlicher Unterschied zur internationalen Umweltpolitik, in der eine wesentlich klarere Trennlinie zwischen Gutwilli- 
gen und weniger Gutwilligen ${ }^{1}$ existiert. Zudem lassen sich reale Aktivitäten on the ground identifizieren, die die Probleme angehen. Dennoch ist das Handlungsfeld zum Ressourcen-Nexus noch weitgehend neu und unstrukturiert. Eine Agenda ist bislang nur in Ansätzen erkennbar; potentielle Akteurskoalitionen oder institutionelle Reformen sind verstreut sichtbar aber noch nicht vernetzt.

\section{Mögliche Konfliktmechanismen}

Rohstoffkonflikte sind nicht neu. Über die Jahrhunderte hinweg haben Rohstoffe aller Art immer Begehrlichkeiten geweckt. Die Entdeckung Amerikas im 15. Jahrhundert und die folgende Eroberung war nicht zuletzt durch die Nachfrage Europas nach Gewürzen, Seide und Gold bestimmt. Die vielen weltweiten Krisen im 17. Jahrhundert, in Europa der Dreißigjährige Krieg 1618-1648, wurden nach dem Historiker Geoffrey Parker (2013) durch Klimaänderungen in dieser Periode forciert. Im Zweiten Weltkrieg war der japanische Angriff auf den US-Luftwaffenstützpunkt Pearl Harbour durch das Erdöl-Embargo motiviert, das die USA gegen Japan in Kraft gesetzt hatten. Auch Nahrungsmittel-Krisen als Auslöser für Revolten, Revolutionen und bewaffnete Konflikte sind nicht neu. Den Revolutionen in Frankreich 1789 und in Russland 1917 gingen spürbare und schockartige Anstiege der Lebensmittelpreise voraus (Oberschall und Seidman 2005).

Natürlich muss man sich vor Verallgemeinerungen ebenso hüten wie vor monokausalen Erklärungen. Die historischen Erfahrungen haben zu vielen erfolgreichen Kooperationsmechanismen geführt. Die Gründung der Europäischen Union und ihrer Vorläufer als Europäische Gemeinschaft für Kohle und Stahl in den fünfziger Jahren des letzten Jahrhunderts ist eine solche Institutionalisierung; ähnliches gilt für die vielen internationalen Wasserabkommen und lokalen Regelungen zur gemeinschaftlichen Landnutzung. Die reale Frage lautet also: Unter welchen Umständen kooperieren Staaten und andere Akteure, unter welchen Bedingungen sind derartige Kooperationsmechanismen wirksam, und unter welchen Bedingungen wählen Staaten und andere Akteure eine Konfliktstrategie oder steigen aus Kooperationsabkommen aus? Welche Konfliktmechanismen entstehen, wenn relevante Akteure nicht kooperieren, welche Eskalationen sind denkbar? Die im Folgenden genannten Faktoren sollten Anlass zur Beunruhigung sein.

Weltweit lässt sich ein Anstieg des Ressourcennationalismus beobachten. Treiber sind die Regierungen in den starken Schwellenländern China, Russland, Brasilien, Indien u. a. m. und deren Rohstoffpolitiken, die jeweils Teil der Sicherheitsstrategie sind (Lee et al. 2012; Mildner 2011; Hilpert und Mildner 2013). Die Enteignung der spanischen Tochterfirma von Repsol in Argentinien 2012 ist nur ein Beispiel unter vielen für die Verlagerung der Machtverhältnisse hin zu den Anbieterländern. Ressourcennationalismus umfasst nicht nur eine einseitige Vertragsaufkündigung und die Nichtanerkennung von Prinzipien offener Märkte und geregelter Außenwirtschaftsbeziehungen, sondern auch die latente und offene Androhung von militärischer Gewalt.

\footnotetext{
${ }^{1}$ Vgl. hierzu die von Volker von Prittwitz 1984 in seinem Buch über Umweltaußenpolitik eingeführten „Helferinteressen“ in der Umweltpolitik.
} 
Als Folge steigen die Risiken bewaffneter Auseinandersetzungen zwischen Staaten auf lokaler Ebene an, vor allem in Krisengebieten im Nahen Osten, in Asien, Afrika und Lateinamerika. Einige Konflikte sind in unserer unmittelbaren Nachbarschaft. Die Entdeckung von Gasvorkommen im südöstlichen Mittelmeerraum und vor den britischen Falklandinseln im Südatlantik, die Wasserkonflikte in Teilen Nordamerikas und nicht zuletzt die aktuellen Konflikte in der Ostukraine und im Irak zeigen die unmittelbaren Risiken für Deutschland und die westlichen Partner. Keiner der regionalen Konflikte wird Deutschland unberührt lassen.

Ein Merkmal von Konflikten, die aus dem Ressourcennationalismus resultieren können, sind asymmetrische Machtbeziehungen zwischen politisch-militärisch starken und schwachen Ländern. Aber beobachtbar sind auch wachsende Konfliktkonstellationen zwischen starken Nachbarn, so zwischen China und Japan, oder Indien und Pakistan.

Weil Migrationen in Krisenregionen zunehmen und nicht zuletzt aus Wasser- und Nahrungsmittelverknappungen sowie illegalen Transporten resultieren, errichten die jeweils stärkeren Nachbarländer Grenzzäune. Die Gesamtlänge und die Bewaffnung dieser Grenzzäune liegt mittlerweile deutlich über den Jahren des Kalten Krieges. Bekannt sind die Grenzzäune zwischen USA und Mexiko, Spanien und Marokko, Israel und Ägypten und Palästina. Weitere Errichtungen unter vielen anderen sind Demarkationen zwischen Saudi Arabien und Jemen, zwischen Indien und Bangladesch, Burma und Pakistan sowie zwischen Botswana und Simbabwe (Jones und Johnson 2014). Während der freie Verkehr von Waren, Kapital und Personen zu den Grundprinzipien der freien Welt gehören, schaffen derartige Errichtungen andere Fakten und sind Ausdruck von Misstrauen gegenüber Kooperationsmechanismen.

Neben potentiellen zwischenstaatlichen Konflikten, deren Treiber Ressourcennationalismus ist, muss man auch die Konfliktmechanismen in fragilen Staaten beobachten. Zugangs- und Verteilungskonflikte vertiefen und verlängern bestehende Bürgerkriege und bewaffnete Auseinandersetzungen. Die Konfliktspirale aus dem Ressourcen-Nexus liegt im Interesse an Einnahmen aus der Ressourcennutzung, die der dauerhaften Finanzierung von bewaffneten Gruppen und organisierter Kriminalität dienen. Diese Aktivitäten gehen über in Terrorismus und Sezessionismus. Länderbeispiele sind die Demokratische Republik Kongo, Jemen und der Sudan. Fragile Regionen können auch in größeren Ländern entstehen, z. B. in Indien (Rasul 2014). In all diesen Ländern vernetzen sich einzelne Rohstoffkonflikte mit Wasser-, Landnutzungs- und Nahrungsmittelkonflikten. Zudem sind internationale Lieferketten mit betroffen, aber auch Mitverursacher der Konflikte.

Die Preisentwicklung wird diese Konflikte voraussichtlich verschärfen. In den kommenden zehn bis zwanzig Jahren sind weitere Nachfragesteigerungen nach natürlichen Ressourcen zu erwarten. Aufgrund einer Vielzahl von Restriktionen ist nicht zu erwarten, dass die Märkte diese Belastungen problemlos meistern werden. Die Preise werden auf hohem Niveau enormen Schwankungen unterworfen sein. Bereits die Preissteigerungen seit dem Jahr 2000 haben die langfristigen Preissenkungen seit dem Ende des Zweiten Weltkriegs ausgeglichen und in die Höhe getrieben. Die künftigen Verknappungen werden für Staaten, Unternehmen und für die Bevölkerung spürbar sein. Investitionen in Ressourceneffizienz wären eine rationale Antwortstrategie. Man muss jedoch beobachten, dass Gewinnerwartungen und Kos- 
tensenkungsdruck auch Anreize zur schnellen Mitnahme bieten, zur Korruption, zur Auslagerung von Folgekosten, und zur Finanzierung illegaler Aktivitäten. Spekulationen mit Rohstoffen werden nicht nur von windigen Händlern an westlichen Börsen betrieben, sondern auch von China und anderen Ländern. Insofern sind es nicht nur Marktverzerrungen, die es zu beachten gilt, sondern auch illegale Aktivitäten und politische Risiken.

Aus dem Ressourcen-Nexus in Kombination mit Governance-Schwächen und mangelndem Kooperationswillen resultieren Konfliktmechanismen, die schwerwiegender und komplexer sind als die bekannten Friktionen früherer Jahre:

- Die globalen Umweltveränderungen wirken sich insbesondere auf das Wasserund Nahrungsmittelangebot aus und sind in Ausmaß, Geschwindigkeit und in ihren Überraschungsmomenten neuartig.

- Neue Akteure - Schwellenländer und staatliche Rohstoffkonzerne - mit politischem und wirtschaftlichem Gewicht sind auf der Weltbühne aktiv und entziehen sich der Atlantikcharta der freien und regelgebundenen Kooperation.

- Der politische Zerfall ganzer Staaten und Regionen führt zu chaotischen und unkalkulierbaren Machtverhältnissen mit internationaler Ausstrahlung.

Die Risikoforschung kennt so genannte Black Swan Events (Taleb 2010), deren Eintreffen nicht als wahrscheinlich angesehen werden muss, die aber durch eine Verkettung von Zufällen entstehen können und hohes Schadenspotenzial haben. Nicht zuletzt die weltweite Finanzkrise hat gezeigt, dass das Erkennen von Einzelrisiken nicht ausschließt, dass ihr Schadenspotenzial unterschätzt wird und deren Verkettung zu weitaus größeren Schäden führen kann. Insofern muss man Analysetools und offene Zukunftsszenarien entwickeln, um auch größere Risiken abschätzen zu können. Was wären solche Großrisiken, die in absehbarer Zeit aus dem RessourcenNexus resultieren könnten? Hier ein Versuch:

- Wichtige Rohstofflieferanten werden Wasser- und Nahrungsmittelkrisen bewältigen müssen und könnten sich sozial und politisch destabilisieren (Bleischwitz et al. 2013). Könnten also wichtige Industrien ernsthafte Lieferprobleme bekommen, weil etwa kritische Materialien nur von wenigen Anbietern hergestellt werden und weil gängige Kritikalitätsanalysen der EU und der USA den Ressourcen-Nexus noch nicht erfassen?

- Was kann in Regionen wie Pakistan, Indien und im Nahen Osten passieren, in Ländern mit Nuklearwaffen, wenn dort Ressourcenkonflikte, politische Krisen und Terrorismus eskalieren? Wie könnten Mechanismen zur Bedarfsplanung, zur Kooperation und zur Kontrolle der geplanten Kernkraftwerke und Staudämme aussehen? Welche machbaren Wege bieten sich für eine grenzüberschreitende Wasser- und Energieversorgung?

- Wie stabil sind die Nahtstellen der Weltwirtschaft für den Transport von Erdöl und Gütern an den Wasserstraßen von Hormuz und Malakka? Was wäre, wenn Piraterie und Terrorismus in einigen Teilregionen der Auftakt für einen Flächenbrand sind, der sich aus Nahrungsmittel- und Wasserkrisen weiter befeuert?

- Ist es auszuschließen, dass Russland, China oder andere Länder ihre Rohstoffstrategien mit militärischen Mitteln verfolgen? Wie reagiert die politische Elite dort, 
wenn die aufstrebende Mittelschicht angesichts steigender Preise für Nahrungsmittel, Energie und Wasser und schmutziger Atemluft rebellisch wird? Könnte Kriegsführung als politisches Ventil gesehen werden, wie etwa Herfried Münkler vermutet, der in einem Interview (Münkler 2014) die Außenpolitik Chinas mit der Vorkriegssituation am Vorabend des Ersten Weltkriegs verglich?

Zusammengefasst verstärkt der Ressourcen-Nexus Konfliktmechanismen des Ressourcennationalismus, des Zerfalls von Staatlichkeit, die Verwundbarkeit von Märkten und das krisenhafte Zusammenwirken von innen- und außenpolitischen Faktoren. Einzel- und zwischenstaatliche Analysen müssen jedoch ergänzt werden durch eine genauere Analyse der Nutzungsplanung für Ressourcen und der Handlungsmuster der jeweiligen Akteure vor Ort.

\section{Kooperationschancen}

Die beschriebenen Konfliktmechanismen bieten erhebliches Konfliktpotenzial, aber auch Kooperationschancen (Bleischwitz 2011, Reder \& Pfeifer 2012). In der Vergangenheit sind viele erfolgreiche internationale Wasserabkommen geschlossen worden. Das Internationale Abkommen zum Schutz der Ozonschicht kann ebenso als Erfolgsfall gelten wie die Arbeit einiger internationaler Institutionen wie beispielsweise die Internationale Atomenergie-Organisation (IAEA). Zudem gibt es ermutigende Kooperationen, beispielsweise zwischen dem wasserarmen Chile und Technologieanbietern aus Israel. Die EU kann sowohl Kompetenzen und Kooperationen anbieten als auch Konditionen für den Zugang zum Binnenmarkt.

Die vermutlich wichtigste Chance liegt in einer Erhöhung der Ressourceneffizienz. Alle wirtschaftlichen Akteure haben ein Interesse daran, Ressourcen so sparsam wie möglich einzusetzen. Potentiale liegen in allen Ländern und sind für viele Akteure erschließbar (Dobbs et al. 2011). Hemmnisse liegen in mangelnden Informationen und einer mangelnden Verknüpfung entlang von Wertschöpfungsketten sowie den beschriebenen internationalen Verzerrungen. Allerdings sollte man bei Ressourcen zwischen Anbieter- und Verbraucherländern differenzieren: Anbieterländer müssten dieses Thema weitgehend neu erschließen und auf ihre Situation im Bergbau und in der Landwirtschaft anwenden, während Verbraucherländer bislang aus guten Gründen bei der verarbeitenden Industrie ansetzen. Zudem sind bisherige Ansätze noch vielfach einzelstoffbezogen und schöpfen die Chancen von mehr systemisch angelegten Öko-Innovationen nicht aus.

Während erste Schritte auf der Ebene einzelner Unternehmen erfolgen können, sind für umfassende Änderungen Kooperationen erforderlich, die andere Marktakteure und die Politik erfordern. Voraussichtlich werden also polyzentrische Formen von Governance erfolgreich sein und Vertiefungen von Public Private Partnerships. Initiativen in dieser Richtung sind vielfach beobachtbar, so etwa die Circular Economy Politik in China, die Ressourceninitiative der Deutschen Gesellschaft für Internationale Zusammenarbeit in Indien, und die Initiative für eine zukunftsfähige Bergbau- und Metallindustrie des Weltwirtschaftsforums (WEF 2014). 
Es ist gegenwärtig offen, in welchen Regionen sich zum Ressourcen-Nexus eher Konfliktmechanismen ergeben werden und wo Kooperationschancen wahrscheinlicher sind. An den Weggabelungen und bei den Entscheidungssituationen zwischen Konflikt- und Kooperationsstrategien wären Frühwarnsysteme hilfreich, für die im Folgenden erste Überlegungen unternommen werden.

\section{Erste Schritte zu einem Frühwarnsystem}

Ein strukturelles Hemmnis besteht im administrativ-selektiven Zuschnitt des Ressourcenmanagements (silo-thinking). Überwiegend werden einzelne Ressourcen behandelt, oder es werden Prioritätenlisten erstellt, ohne die Wechselwirkungen hinreichend in Betracht zu ziehen. Ein Schlüssel liegt darin, von vermeintlichen „Rohstoffinteressen“ überzugehen zu einer Analyse von Bedarfssystemen zur Bedürfnisbefriedigung und zur Rolle von Staatlichkeit und Märkten. Das vitale Interesse an Energie, Wasser- und Nahrungsmitteln und die erkennbare Aufmerksamkeit für den Nexus zwischen diesen Ressourcen ist daher zu begrüßen. Konkret gesagt ließe sich der Ressourcen-Nexus für Versorgungssysteme im öffentlichen Interesse abschätzen, sowohl auf der lokalen als auch auf der nationalen Ebene. Dies würde eine Analyse der jeweils erforderlichen Inputs umfassen und sollte dazu führen, dass Ressourcennutzungs-Koeffizienten zu wesentlichen Produktgruppen und Infrastruktursystemen erstellt werden (z. B. Wasserinanspruchnahme des Kraftwerks XY). Auf diese Weise könnten Unternehmen und Märkte pro-aktiv arbeiten.

Die bisherigen Erfahrungen haben gezeigt, dass die relative Abhängigkeit und die Geschwindigkeit von Veränderungen ein Maßstab für Verwundbarkeiten sind. Liegt beispielsweise die Importabhängigkeit oder liegt die Geschwindigkeit einer Nachfrageänderung höher als im internationalen Durchschnitt, wächst die Verwundbarkeit. Entscheidend aus Sicht des Ressourcen-Nexus sind die Miterfassung der jeweils anderen Ressourcen und eine Analyse der Situation in den Herkunftsregionen. Man benötigt also international vergleichende Maßstäbe.

Ein internationaler Informationsaustausch und Transparenzregeln sind ein Schlüssel für verbesserte Kooperationen. Vorhandene Initiativen sind Schritte in die richtige Richtung, aber fragmentiert und nicht annähernd auf die oben beschriebenen Erfordernisse ausgerichtet (Extractive Industries Transparency Initiative, Carbon Disclosure Project, Green Growth Knowledge Platform etc.).

Es bedarf eines systematischen Monitorings von Preisen und Mengen globaler Rohstoff- und Ressourcenflüsse. Die internationalen industrienahen Studiengruppen für Kupfer, Stahl usw. sollten ebenso aktiviert werden wie die geologischen Dienste und Umweltnetzwerke ${ }^{2}$ und die genannten internationalen Organisationen. Auf Basis eines neuen offenen Informationsportals sollten Szenarien zum künftigen Ressourcenverbrauch erstellt werden, die bislang erst ansatzweise vorliegen. Einige dieser Szenarien sollten auch Krisenszenarien sein. Entsprechende Modellierungen könnten

\footnotetext{
${ }^{2}$ Vgl. Daten zu Materialflüssen: www.worldmrio.com, www.materialflows.net. Zu Wasserthemen ist die World Water Week einschlägig.
} 
an der Schnittstelle zwischen Umweltanalysen und makro-ökonomischer Modellierung ansetzen und sollten sozio-politische Szenarien einbeziehen.

Wichtig sind zukunftsgerichtete Akteursanalysen. Die beginnenden Herkunftsnachweise und finanziellen Transparenzregelungen für einige Rohstoffe (Bleischwitz 2014) müssen flankiert werden durch Analysen von Schlüsselländern, ihre zwischenstaatlichen Beziehungen und Konfliktmechanismen in Krisenregionen. Die traditionelle internationale Politikanalyse ist entsprechend zu vertiefen; sie muss auch um Analysekomponenten erweitert werden, die innerstaatliche, soziale und marktbezogene Dynamiken erfassen.

Politisch ergeben sich Chancen, wenn vorhandene Energie- und Rohstoffpartnerschaften systematisch in den Bereichen integriertes Landnutzungs- und Umweltmanagement und Ressourceneffizienz erweitert werden.

Die bisherigen Ansätze in der europäischen Rohstoffinitiative greifen deutlich zu kurz. Eine europäische ressourcenbezogene Außen- und Sicherheitspolitik ist ansatzweise erkennbar, wird aber bislang zu zögerlich angegangen. Neben einer Stärkung der Politik in Deutschland und in der EU müssen schrittweise internationale Netzwerke und letztlich auch Institutionen gestärkt und aufgebaut werden. Unterhalb der Ebenen der G7 (ehemals G8) und G20 könnte ein erstes Ressourcenforum eingerichtet werden, das die wichtigsten Länder umfasst; der Chatham House Bericht spricht sich für die Gründung einer $R 30$ aus (Lee at al. 2012). Das Internationale Ressourcenpanel des Umweltprogramms der Vereinten Nationen könnte ein Mandat erhalten für einen regelmäßigen Statusbericht zum Ressourcen-Nexus. In weiteren Schritten könnte man ein Multi-Stakeholder-Forum zum Ressourcen-Nexus gründen und - warum nicht! - eine internationale Ressourcenagentur mit einem vergleichbaren Mandat wie die Internationale Energieagentur (IEA).

\section{Schlussfolgerungen}

Der Ressourcen-Nexus erzeugt einen hohen Handlungsdruck, indem er vorhandene latente Konflikte vertieft und neue Eskalationsrisiken schafft. In extremen Fällen können neue zwischenstaatliche Konflikte entstehen. Für die dort lebenden Menschen hat dies dramatische Folgen. Für alle Märkte werden die Folgen spürbar sein. In der Zukunft dürften sich diese Risiken verstärken. Insofern besteht Analyse- und Handlungsbedarf für die Außen- und Sicherheitspolitik. Unser Beitrag identifiziert Konfliktmechanismen und benennt einige anfällige Regionen.

Diese Risiken bieten jedoch auch Anreize zu Kooperationen. Insbesondere die Chancen einer erhöhten Ressourceneffizienz müssen stärker international vernetzt werden. International koordinierte Anstrengungen zur Ressourceneffizienz sollten über einzelne gewerbliche Unternehmen hinausgehen und die wesentlichen Wertschöpfungsketten sowie auch die Bereiche Land- und Wasserwirtschaft sowie Bergbau umfassen. Angesichts der vielen aktuellen Krisen und Konflikte ist es günstig zu sehen, dass diese Schritte jetzt machbar sind und viele Akteure umfassen können. Mittelfristig können Einnahmen aus dem Ressourcenbereich für zukunftsfähige Investitionen genutzt werden. 
Die Frage bleibt jedoch, wie man mit dem strategischen Verhalten ressourcennationalistischer Akteure umgeht. Erste machbare Steuerungsmöglichkeiten sind auch hier erkennbar. Man sollte jetzt international schrittweise ein Wissensmanagement einrichten, das verbesserte Informationen institutionalisiert und ihre Umsetzung erleichtert. Bilaterale und multilaterale Energie- und Rohstoffpartnerschaften können zielgerichtet zu Abkommen eines nachhaltigen Ressourcenmanagements erweitert werden. Die Vorschläge der Oxford Martin Commission (2013) zur Rolle neuer Koalitionen sind im Ressourcenbereich besonders interessant, da sie themen- und regionalspezifisch mit Unternehmen, Zivilgesellschaft und NGOs an vorhandene Initiativen anknüpfen und umgesetzt werden können. Insofern gehen die Möglichkeiten über eine Umweltaußenpolitik hinaus.

Transparenzbestrebungen für extraktive Industrien werden politisch in den USA und in der EU eingefordert. Deutschland hat im Bereich der internationalen Rohstoffpartnerschaften und Ressourceneffizienz und mit der Etablierung von IRENA, der Internationalen Agentur für Erneuerbare Energien, erste gute Schritte unternommen. Jetzt sollte es an der Zeit sein, eine größere internationale Initiative zum RessourcenNexus zu initiieren. So kann schrittweise ein internationales Governance-System für ein nachhaltiges Ressourcenmanagement aufgebaut werden.

Open Access Dieser Artikel unterliegt den Bedingungen der Creative Commons Attribution Noncommercial License. Dadurch sind die nichtkommerzielle Nutzung, Verteilung und Reproduktion erlaubt, sofern der/die Originalautor/en und die Quelle angegeben sind.

\section{Literatur}

Ackerman, F., \& Fisher, J. (2013). Is there a water-energy nexus in electricity generation? Long-term scenarios for the western United States. Energy Policy, 59(0), 235-241.

Andrews-Speed, C. P., Bleischwitz, R., Boersma, T., Johnson, C., Kemp, G., \& VanDeever, S. D. (2012). The global resource nexus. The struggle for land, energy, food, and minerals. Report of the Transatlantic Academy, Washingon D.C.

Andrews-Speed, C. P., Bleischwitz, R., Boersma, T., Johnson, C., Kemp, G., \& VanDeveer, S. D. (2014). Want, waste or war? The global resource nexus and the struggle for land, energy, food, water and minerals. Abingdon: Routledge Publisher.

Bazilian, M, Rogner, H, Howells, M, Hermann, S., Arent, D., Gielen, D., Steduto, P., Mueller, A., Komor, P., Tol, R. S. J., \& Yumkella, K. K. (2011). Considering the energy, water and food nexus: Towards an integrated modeling approach. Energy Policy, 39(12), 7896-7906.

Bleischwitz, R. (2011). Neue Governance-Mechanismen für ein global nachhaltiges Ressourcenmanagement. Zeitschrift für Außen- und Sicherheitspolitik, 4(3), 399-410.

Bleischwitz, R. (2014). Transparency in the extractive industries: Time to ask for more. Global Environmental Politics, 14(4), (i. E.).

Bleischwitz, R., Johnson, C., \& Dozler, M. (2013). Re-Assessing resource dependency and criticality. Linking future food and water stress with global resource supply vulnerabilities for foresight analysis. European Journal for Futures Research, 2(1), 1-12.

Breisinger, C., Olivier, E., \& Perrihan, A.-R. (2011). Economics of the Arab Awakening. From revolution to transformation and food security, IFPRI Policy Brief 18. International Food Policy Research Institute. http://www.ifpri.org/sites/default/files/publications/bp018.pdf. Zugegriffen: 14. Okt. 2014. 
Dobbs, R., Oppenheim, J., Thompson, F., Brinkman, M., \& Zornes, M. (2011). Resource revolution: Meeting the world's energy, materials, food and water needs. McKinsey Global Institute. http://www. mckinsey.com/ /media/McKinsey/dotcom/Insights\%20and\%20pubs/MGI/Research/Resource\%20 Markets/Resource\%20revolution/MGI_Resource_revolution_full_report.ashx. Zugegriffen: 14. Okt. 2014.

European Commission. (2012) Confronting scarcity: Managing water, energy and land for inclusive and sustainable growth. The 2011/2012 European Report on Development. http://erd-report.eu/erd/ report_2011/documents/erd_report\%202011_en_lowdef.pdf. Zugegriffen: 14. Okt. 2014.

Ghosh, A. (2013). India's resource nexus: priorities for action. http://www.livemint.com/Opinion/ zAOvm6gwBKa6Bzr9DfSyxN/Indias-resource-nexus-priorities-for-action.html. Zugegriffen: 14. Okt. 2014.

Giampietro, M., Aspinall, R. J., Bukkens, S. G. F., Cadillo Benalcazar, J., Diaz-Maurin, F., Flammini, A., Gomiero, T., Kovacic, Z., Madrid, C., Ramos-Martín, J., \& Serrano-Tovar, T. (2013). An innovative accounting framework for the food-energy-water nexus. Application of the MuSIASEM approach to three case studies. FAO Environmental and Natural Resources Working Paper, 56.

Giurco, D., McLellan, B., Franks, D. M., Nansai, K., \& Prior, T. (2014). Responsible mineral and energy futures: views at the nexus. Journal of Cleaner Production, (i. E.).

Hilpert, H.-G., \& Mildner, S.-A. (2013). Nationale Alleingänge oder internationale Kooperation? Analyse und Vergleich der Rohstoffstrategien der G20-Staaten. SWP-Studie S 01. Berlin: Stiftung Wissenschaft und Politik (SWP) \& Hannover: Bundesanstalt für Geowissenschaften und Rohstoffe.

Hoff, H. (2011). Understanding the nexus. Background paper for the Bonn 2011 Conference: The water, energy and food security nexus. Stockholm Environment Institute, Stockholm. http://www.waterenergy-food.org/documents/understanding_the_nexus.pdf. Zugegriffen: 14. Okt. 2014.

Jones, R., \& Johnson, C. (Hrsg.). (2014). Placing the border in everyday life. Farnham: Ashgate.

Lawford, R., Bogardi, J., Marx, S., Jain, S., Pahl-Wostl, C., Knüppe, K., Ringler, C., Lansigan, F., \& Meza, F. (2013). Basin perspectives on the water-energy-food security nexus. Aquatic and marine systems, $5(6), 607-616$.

Lee, B., Preston, F., Kooroshy, J., Bailey, R., \& Lahn, G. (2012). Resources futures. A Chatham house report. London: Royal Institute of International Affairs.

Mildner, S.-A. (Hrsg.). (2011). Konfliktrisiko Rohstoffe? Herausforderungen und Chancen im Umgang mit knappen Ressourcen. SWP-Studie S 05. Berlin: Stiftung Wissenschaft und Politik (SWP), Deutsches Institut für Internationale Politik und Sicherheit.

Mo, W., \& Zhang, Q., (2013). Energy-nutrients-water nexus: Integrated resource recovery in municipal wastewater treatment plants. Journal of Environmental Management, 127, 255-267.

Mukherji, A. (2007) The energy-irrigation nexus and its impact on groundwater markets in eastern IndoGangetic basin: Evidence from West Bengal, India. Energy Policy, 35(12), 6413-6430.

Münkler, H. (2014). Zeitraffer eines Jahrhunderts. Frankfurter Allgemeine Zeitung. http://www.faz.net/ aktuell/feuilleton/debatten/2.3223/herfried-muenkler-ueber-den-ersten-weltkrieg-zeitraffer-einesjahrhunderts-12766254.html. Zugegriffen: 14. Okt. 2014.

NIC - National Intelligence Council (2012). Global water security. ICA 2012-08, 2 February 2012. http:// fas.org/irp/nic/water.pdf. Zugegriffen: 14. Okt. 2014.

Oberschall, A., \& Seidman, M. (2005). Food coercion in revolution and civil war: Who wins and how they do it. Comparative Studies in Society and History, 47(2), 372-402.

Oxford Martin Commission (2013). Now for the long term. The Report of the Oxford Martin Commission for Future Generations, Oxford Martin School, Oxford University 2013.

Parker, G. (2013). Global crises. War, climate change and catastrophe in the seventeenth century. New Haven: Yale University Press.

Rasul, G. (2014). Food, water, and energy security in South Asia: A nexus perspective from the Hindu Kush Himalayan region. Environmental Science \& Policy, 39, 35-48.

Reder, M., \& Pfeifer, H. (Hrsg.). (2012). Kampf um Ressourcen. Weltordnung zwischen Konkurrenz und Kooperation (Globale Solidarität - Schritte zu einer neuen Weltkultur, Bd. 22). Stuttgart: Kohlhammer.

Ringler, C., Bhaduri, A., \& Lawford, R. (2013). The nexus across water, energy, land and food (WELF): potential for improved resource use efficiency? Aquatic and marine systems, 5(6), 617-624. 
Rockström, J., Steffen, W., Noone, K., Persson, A., Chapin III, F. S., Lambin, E. F., Lenton, T. M, Scheffer, M., Folke, C., Schellnhuber, H. J., Nykvist, B., de Wit, C. A., Hughes, T., van der Leeuw, S., Rodhe, H., Sörlin, S., Snyder, P. K., Costanza, R., Svedin, U., Falkenmark, M., Karlberg, L., Corell, R. W., Fabry, V. J., Hansen, J., Walker, B., Liverman, D., Richardson, K., Crutzen, P., \& Foley, J. A. (2009). A safe operating space for humanity. Nature, 461, 472-475.

Siddiqi, A., \& Anadon, L. D. (2011). The water-energy nexus in Middle East and North Africa. Energy Policy, 39(8), 4529-4540.

Slingerland, S., Prins, A. G., Manders, T., Lucas, P., Hilderink, H., \& Kok, M. (2011). Scarcity in a sea of plenty? Global resource scarcities and policies in the European Union and the Netherlands. Den Haag: PBL - Netherlands Environmental Assessment Agency.

Steffen, W., Persson, A., Deutsch, L., Zalasiewicz, J., Williams, M., Richardson, K., Crumley, C., Crutzen, P., Folke, C., Gordon, L., Molina, M., Ramanathan, V., Rockström, J., Scheffer, M., Schellnhuber, H. J., \& Svedin, U. (2011). The anthropocene: From global change to planetary stewardship. AMBIO, 40(7), 739-761.

Sternberg, T. (2012). Chinese drought, bread and the Arab Spring. Applied Geography, 34, 519-524.

Taleb, N. N. (2010). The black swan: The impact of the highly improbable. 2. Aufl. New York: Random House Trade Paperbacks.

UNESCAP - United Nations Economic and Social Commission for Asia and the Pacific. (2013). The status of the water-food-energy nexus in Asia and the Pacific. Discussion Paper.

WBCSD - World Business Council for Sustainable Development. (2012). Pathway, Water, food and energy nexus. Executive brief, October 2012. Genf: WBCSD.

WEF - World Economic Forum (2014). Scoping paper: Mining and metals in a sustainable world. Genf: WEF. 